\title{
El rol de la ética en los estilos de liderazgo
}

\section{The role of ethics in leadership styles}

Santiago Nájera, Ph.D. Candidate

Universidad Central del Ecuador, Ecuador

Autor para correspondencia: snajera@pucp.pe

Fecha de recepción: 26 de Febrero de 2016 - Fecha de aceptación: 17 de Mayo de 2016

\section{Resumen}

La moral es fundamentalmente relacionada con el efecto de las acciones propias sobre el resultado tanto propio como en los otros. De aquí que el liderazgo ético comprende más que el comportamiento ético o no, comprende un liderazgo efectivo, con el objetivo de lograr en el largo plazo el éxito de la organización y porque no el éxito y mantenimiento del modelo de la organización

Palabras claves: ética; liderazgo; negocios

\begin{abstract}
Morality is fundamentally related to the effect of one's actions on both their own and the other result. Hence ethical leadership involves more than ethical behavior or not, comprising an effective leadership, with the objective of achieving long-term success of the organization and because no success and maintaining the model of the organization
\end{abstract}

Key words: ethics; leadership; business 


\section{Introducción}

Ética es en esencia el estudio de los estándares que determinan todos aquellos comportamientos que son considerados como buenos y cuales comportamientos puedes ser clasificado como malos, existiendo por parte de los filósofos un sin número de teorías al respecto de este tema, a pesar de los distintos puntos de vista al respecto algo es constante en todos los casos, la moral es fundamentalmente relacionada con el efecto de las acciones propias sobre el resultado tanto propio como en los otros. De aquí que el liderazgo ético comprende más que el comportamiento ético o no, comprende un liderazgo efectivo, con el objetivo de lograr en el largo plazo el éxito de la organización y porque no el éxito y mantenimiento del modelo de la organización, siendo el comportamiento ético una condición sine qua non para lograr las condiciones necesarias para establecer una organización con fundamentos éticos. De aquí la importancia de la ética en las organizaciones y por tanto el de medir la integridad percibida del líder, lo cual es posible mediante el uso de distintas herramientas(Craig \& Gustafson, 1998), entre las cuales se encuentra el del Perceived Leader Integrity Scale (PLIS), de aquí que en lo relacionado al comportamiento de los lideres desde el punto de vista ético se busca analizar dos factores fundamentales, el primero es la integridad puesto que este factor está relacionado con la confianza en los lideres, y el segundo es el comportamiento egoísta (Morgan, 1989). Existen en ética empresarial dos grandes corrientes frecuentemente utilizada deontología and teleológica(Aronson, 2001), las cuales se entienden de la siguiente forma.

Teoría deontológica, se puede describir como el estudio de las obligaciones morales, es así que el comportamiento moral es independiente del resultado por tanto no depende de producir el máximo beneficio, puesto que algunos de los posibles resultados son cuestionables al momento de tomar la decisión de actuar (Helms \& Hutchins, 1992). Existen dos categorías principales en la teoría deontológica: reglas deontológicas y actos deontológicos. Las reglas deontológicas, indican que ante cualquier tipo de circunstancias los individuos deben de seguir un grupo predeterminado de reglas y estándares, de aquí que un comportamiento se lo no define como ético no en relación con las consecuencias de la acción, sino en comparación con las reglas (Rallapalli, Vitell, \& Barnes, 1998), de aquí que un juicio ético depende de un principio general y estos principios a su vez dependen de una serie particular de guías, las cuales indican como debe de comportarse un individuo en cierto tipo de condiciones (Frankena, 1973). Los actos deontológicos, son aquellos que indica que las personas actúan de manera ética de acuerdo a ciertas normas pre establecidas pero que están limitadas a cierto comportamiento, lo que implica que puede existir excepciones a la regla (Rallapalli et al., 1998),de aquí que los individuos están obligados a actuar de cierta manera dada puesto existe la obligación de considerar los derechos y dignidad sin importar la consecuencia, de aquí que el valor moral se encuentra en la propia acción (White, 1988) y no en el resultado que esta pueda llegar a producir tanto a la persona como a la sociedad.

Teorías teleológicas, son aquellas que percibe como criterio de éticamente correcto al valor que se crea, es decir un acto es moral si produce un mayor grado de bien sobre el mal, sobre cualquier otra alternativa, y es inmoral si no lo hiciera (Frankena, 1973). Dentro de las teorías teleológicas existen varias clasificaciones, siendo las más importantes y utilizadas: el egoísmo ético, acciones utilitarias y reglas utilitarias. En cuanto al egoísmo ético, se puede decir que el individuo considera su acto moral o inmoral dependiendo de la probabilidad de lograr 
objetivos personales (Rallapalli et al., 1998), de aquí que cualquier otro resultado es considerado irrelevante, es posible adicionalmente que en el egoísmo ético se considere los intereses de los otros pero no como objetivo principal, puesto los otros son vistos como medios por los cuales el individuo puede maximizar sus beneficios personales (Shaw \& Post, 1993). Por otra parte cuando nos referimos al utilitarismo, podemos decir que este es la agregación de dos principios (Quinton, 1988), al principio secuencialismo que comprende que un acto es bueno o malo en función de las consecuencias que genere este acto, y el principio hedonista mismo que valora a todos los actos que generan placer como buenos, mientras que aquellos que generan dolor como actos malos, de aquí que Bentham(1879) señala que lo correcto de una acción es determinado por su contribución a la felicidad de todos los afectado por dicha acción. Es así que al hablar de acciones utilitarias, nos referimos que una acción es correcta u obligatoria en función del principio de utilidad, es decir que se selecciona en comparación de que acción genera el máximo nivel de bienestar total frente a otras. Por otra parte las regla utilitaristas, nos indican que una persona debe actuar en un modo tal que genere el mayor grado de bienestar al mayor número de personas posible (Rallapalli et al., 1998), estas reglas deben de asegurar el resultado más favorable para todos, de aquí que una acción es considerada moralmente correcta puesto tiene un resultado positivo en una situación específica, puesto que dichas acciones deben ser realizadas frecuentemente de manera rápida que imposibilitan la valoración de los resultados posibles a ser obtenidos, es importante contar con un grupo de reglas de actuación (Quinton, 1988), las cuales pueden ser escogidas, modificadas y remplazadas en función de la máxima utilidad.

Tradicionalmente se ha considerado que tanto la teoría deontológica como la teoría teológica son opuestas entre si y mutuamente excluyentes, no obstante algunos autores señalan que las persona no son deontológicas o teológicas en sus puntos de vista morales y que en si ambas teorías pueden llegar a ser complementarias (Brady, 1985; Woller, 1998), de aquí que la mejor forma de resolver problemas éticos es mediante el uso de ambos puntos de vista de manera simultánea, puesto los seres humanos se encuentran motivados por ciertos principios de que es bueno y que es malo, y al mismo tiempo por las consecuencias que sus acciones puedan generar.

\section{Liderazgo ético}

Tradicionalmente se ha definido a los líderes transformacionales como aquellos que influencia a sus seguidores en termino morales, mientras que a los líderes transaccionales y directivos como poco éticos asociados con bajos niveles de desarrollo moral (Kuhnert \& Lewis, 1987; Petrick \& Quinn, 1997), no obstante se debe tener en cuenta que el nivel de desarrollo moral de una persona no determina el tipo de liderazgo, sino más bien que tan ética es la persona, de aquí que el factor determinante del comportamiento ético de un líder es el nivel de desarrollo moral o altruismo, siendo el altruismo la consideración por el bienestar de los otro sin considerar el costo que esto tenga para uno mismo. Los tipos de liderazgo se ven generalmente asociados con comportamientos éticos claramente definidos.

Liderazgo directivo, su actuación moral depende de la probabilidad de lograr alcanzar primero sus objetivos personales y después serán tomado en cuenta los intereses de sus subordinados, siendo los posibles estilos de estos líderes: (a) benevolente autocrático, el líder decide por los otros pero pensando genuinamente en los mejores intereses del resto, (b) consultativo, es aquel que escucha a sus subordinados pero finalmente toma la decisión solo, (c) 
participativo, alienta la participación de sus subordinados en la toma de decisiones, haciendo que estos contribuyan con ideas y soluciones, pero se reserva el derecho de tomar la decisión final, (d) consenso, es un líder similar al líder participativo pero no se reserva el derecho a tomar la decisión final, (e) autocrático despótico, es aquel que distorsiona la misión y objetivos de la organización, abusando de los recursos existente en pro de maximizar sus beneficios propios., y (d) laissez faire, no se lo puede considerar como liderazgo puesto no enfoca sus esfuerzos en función de los intereses de la organización y se contenta con cubrir sus propias necesidades sin importarle las actividades de sus subordinados.

Liderazgo transaccional, su actuación se da en función de un intercambio con sus seguidores, siendo en gran medida sus acciones del tipo utilitaristas al enfocar sus esfuerzos en la obtención del mayor grado de beneficio posible, siendo las posibles características de estos líderes: (a) recompensa contingente, aquel que tiene un alto nivel de desarrollo ético y que busca que la negociación con el subordinado sea realizada de buena fe, (b) gestión activa por excepción, el líder buscara evitar desviaciones de los estándares establecidos mediante continuo control caracterizado por valores éticos, y (c) gestión pasiva por excepción, el líder solo tiende a actuar al momento que se ha generada una desviación de los estándares, no obstante su actuación será bajo preocupación por el bienestar de los otros. Es posible que dentro del liderazgo transaccional aquellos lideres con un bajo desarrollo de comportamiento moral tiendan a ver a sus subordinados únicamente como recursos que les permitirán alcanzar sus metas personales, utilizando su poder para ajustar reglas y procesos de manera que estas les sirvan para servir sus propios interés, en el caso de líderes con bajo nivel de desarrollo moral su comportamiento se asemeja al de laissez faire.

Liderazgo transformacional, son aquellos líderes con un altos valores e ideales los auténticos líderes carismáticos-transformacionales actúan con genuino interés por el beneficio de sus seguidores, son éticos por naturaleza y tienden a actuar guiados por un conjunto de normas morales y un alta preocupación por hacer las cosas correctas, este tipo de líderes demuestran liderazgo ético cuando son guiados por altruismo, buscando guiar a sus subordinados mediante el empoderamiento antes que el control. Generalmente los lideres transformacionales son aquellos que ponen el bienestar de los otros antes que el bienestar propio (Bass \& Steidlmeier, 1999), interesados principalmente por el bienestar de la comunidad y de la organización como un todo, los rasgos característicos de estos tipos de líderes están dados por: (a) influencia idealizadora, altos valores morales en pro de un mejor futuro para todo el grupo, (b) inspiración motivadora, enfatizan las mejores cualidades de las personas del grupo, (c) estimulación ética, implantación de visión y estrategia en un espíritu de apertura y cooperación entre las partes, y (d) consideración individual, comportamiento altruista por naturaleza, dando énfasis al crecimiento de sus seguidores y no únicamente al crecimiento personal. No obstante los líderes transformacionales artificiales son aquellos con comportamiento narcisista y egoísta que expresan exagerada preocupación solo en los beneficios personales (Conger \& Kanungo, 1988), este tipo de líderes busca poder y mantener su estatus, manipulando a sus seguidores y haciendo que estos dependan de él, por lo cual no dudaran de tomar ventaja de los otros. Cuando estos tipos de líderes asumen el poder existe gran riesgo de que estos usen dicho poder en función de sus propios intereses a costa de sus subordinados y la organización.

\section{Conclusiones}


Es importante que las organizaciones al igual que las personas, comiencen a darse cuenta que en la actualidad sus acciones tienen cada vez mayor repercusión en lo que pasa en su entorno, de aquí que el pensamiento unidimensional enfocado netamente al beneficio común puede llegar a tener repercusiones negativas no solamente al entorno, sino adicionalmente a la misma organización, motivo por el cual cada vez incrementa la necesidad de que las organizaciones manejen sus negocios con una conducta ética, de aquí la necesidad de contar con buenos líderes los cuales deben contar con características de efectividad y moralidad, haciendo que las organizaciones con orientación económica no conduzcan su estrategia a expensas de otros, logrando que la estrategia de la empresa logre combinar el "Yo" y el "Nosotros" en la toma de decisiones. Por tanto es importante contar con esos líderes con características inspiradoras, aquellos lideres visionarios que hagan que el resto de la organización se encamine a estándares mayores, que permitan que la empresa se desarrolle al igual que cada uno de sus miembros en conjunto con la sociedad y el entorno en cual se desempeña la organización, este tipo de líderes son los lideres transformacionales (Bass \& Steidlmeier, 1999).

\section{Bibliografía}

Aronson, E. (2001). Integrating leadership styles and ethical perspectives. Canadian Journal of Administrative Sciences/Revue Canadienne des Sciences de l'Administration, 18(4), 244-256.

Bass, B. M., \& Steidlmeier, P. (1999). Ethics, character, and authentic transformational leadership behavior. The Leadership Quarterly, 10(2), 181-217.

Bentham, J. (1879). An introduction to the principles of morals and legislation: Clarendon Press.

Brady, F. N. (1985). A Janus-headed model of ethical theory: Looking two ways at business/society issues. Academy of Management Review, 10(3), 568-576.

Conger, J. A., \& Kanungo, R. N. (1988). Charismatic leadership: The elusive factor in organizational effectiveness: Jossey-Bass.

Craig, S. B., \& Gustafson, S. B. (1998). Perceived leader integrity scale: An instrument for assessing employee perceptions of leader integrity. The Leadership Quarterly, 9(2), 127-145.

Frankena, W. K. (1973). Ethics, : Prentice Hall, Englewood Cliffs, NJ.

Helms, M. M., \& Hutchins, B. A. (1992). Poor quality products: is their production unethical? Management decision, 30(5).

Kuhnert, K. W., \& Lewis, P. (1987). Transactional and transformational leadership: A constructive/developmental analysis. Academy of Management Review, 12(4), 648-657.

Morgan, R. B. (1989). Reliability and validity of a factor analytically derived measure of leadership behavior and characteristics. Educational and Psychological Measurement, 49(4), 911-919.

Petrick, J. A., \& Quinn, J. F. (1997). Management ethics: Integrity at work (Vol. 6): Sage.

Quinton, A. (1988). Utilitarian ethics.

Rallapalli, K., Vitell, S., \& Barnes, J. (1998). The influence of norms on ethical judgments and intentions: An empirical study of marketing professionals. Journal of Business Research, 43(3), 157-168.

Shaw, B., \& Post, F. R. (1993). A moral basis for corporate philanthropy. Journal of Business Ethics, 12(10), 745-751.

White, T. I. (1988). Right and wrong: A brief guide to understanding ethics: Prentice-Hall Englewood Cliffs, NJ.

Woller, G. M. (1998). Toward a reconciliation of the bureaucratic and democratic ethos. Administration \& society, 30(1), 85-109. 